\title{
豌豆乙酰羟酸还原异构酶基因结构、蛋白 活性分析及其调控机制研究
}

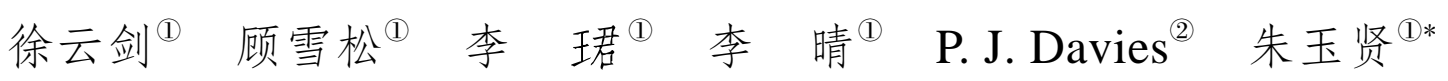

(1)北京大学蛋白质工程及植物基因工程国家重点实验室, 北京 100871; 2Department of Plant Biology, Cornell University, Ithaca, NY 14853, USA)

\begin{abstract}
摘要用 PCR 方法获得了 G2 踠豆 AAIR 基因的核 DNA 序列, 分析表明该基因由 8 个内含子和 9 个外显子组成, 其中 3 个内含子带有特征性的富 $\mathrm{A} / \mathrm{T}$ 内源启动子区. 分子 杂交试验表明, 短日照条件下 G2 豌豆中 AAIR 的表达不受生殖生长的影响, 开花前后 都维持较高水平的表达. 长日照条件下, 植株开花后 AAIR 的表达水平迅速下降. AAIR 基因表达强度的变化与豌豆乙酰羟酸还原异构酶的活性变化规律相一致. 用乙酰羟酸 还原异构酶缺陷型大肠杆菌做功能互补实验发现, 带有踠豆 AAIR cDNA 的细菌能够在 缺少分枝氨基酸的 M9 培养基上生长, 表明该基因产物确实具有乙酰羟酸还原异构酶活 性. 进一步的研究表明, 豌豆 $A A I R$ 基因产物能显著提高野生型大肠杆菌合成分枝氨基 酸的能力. 凝胶阻滞实验表明, AAIR 基因的内含子中的富 $\mathrm{A} / \mathrm{T}$ 区能与豇豆核蛋白提取物 发生特异性结合. 该蛋白在不衰老的短日照 G2 踠豆顶芽中一直保持稳定表达, 但在迅 速衰老的长日照豌豆顶芽中, 开花后该蛋白就迅速降低直至完全消失. 因此, AAIR 基因 内源启动子区与核蛋白的特异性结合可能是调控该基因表达的重要机制.
\end{abstract}

\section{关键词 AAIR 基因 $\mathrm{G} 2$ 豌豆 富 $\mathrm{A} / \mathrm{T}$ 区 酶活性 衰老}

运用 cDNA 代表群差示分析技术, 朱玉贤等人 ${ }^{[1]}$ 从 G2 踠豆中克隆到了一个受赤霉素诱导 表达的 cDNA, 其编码产物与许多生物中的分枝氨基酸合成过程中的乙酰羟酸还原异构酶 (AAIR, acetohydroxy acid isomeroreductase, 又称 ketol-acid reductoisomerase, EC1.1.1.86)有很 高的同源性, 并表现出催化 AHB(2-aceto-2-hydroxybutyrate)和 NADPH 的反应活性, 因此认为 该基因编码了踠豆的乙酰羟酸还原异构酶 ${ }^{[2]}$. AAIR 是细菌、真菌和植物中的分枝氨基酸(Val, Ile 和 Leu)生物合成途径中一个很重要的酶 ${ }^{[3]}$, 虽然 Ile 和 Val 的生物合成途径是两个相对独 立的反应过程，乙酰羟酸还原异构酶同时催化这两条途径的第二步反应 ${ }^{[3 \sim 5]}$.

除了合成分枝氨基酸外, 乙酰差酸还原异构酶还可能与细胞分裂有一定关系 ${ }^{[6,7]}$. 酵母中 该酶定位在细胞的线粒体中, 被认为在提高线粒体 DNA 的稳定性方面起重要作用 ${ }^{[8 \sim 10]}$. 此外, 细胞衰老过程中, 膜结构变化很大, 脂质过氧化、膜蛋白降解、膜通透性增加, 最终导致膜完 整性的丧失. 分枝氨基酸生物合成中的许多中间产物, 特别是支链酮酸, 是支链脂肪酸合成的 前体, 后者是膜脂类的重要组成部分 ${ }^{[11]}$. 本文对踠豆 AAIR 基因的结构、酶活性变化及其表达 
调控模式做了进一步研究, 不但发现该基因产物确实具有乙酰羟酸还原异构酶的活性, 能催 化分枝氨基酸的生物合成, 而且发现 $A A I R$ 核基因的内含子中含有一些与核蛋白特异结合的富 含 $\mathrm{A} / \mathrm{T}$ 区, $A A I R$ 基因的表达规律与 $\mathrm{G} 2$ 豌豆的衰老进程有紧密联系.

\section{1 材料与方法}

\section{1 植物材料及培养}

G2 踠豆 (Pisum sativum L.) 种子由本实验室保存. 种子播种于温室内 $4 \mathrm{dm}^{3}$ 塑料盆中. 平均 光照强度为 $300 \mu \mathrm{mol} /\left(\mathrm{m}^{2} \cdot \mathrm{s}\right)$, 光照时温度为 $23^{\circ} \mathrm{C}$, 黑暗时温度为 $19^{\circ} \mathrm{C}$. 短日照为每 24 小时 9 $\mathrm{h}$ 光照和 $15 \mathrm{~h}$ 黑暗, 长日照为每 24 小时 $18 \mathrm{~h}$ 光照和 $6 \mathrm{~h}$ 黑暗.

\section{2 酶和生化试剂}

各种限制性内切酶、T4 DNA 连接酶、Klenow 酶、IPTG 均购自 Promega 公司. Ex taq DNA polymerase 为 TaKaRa 公司产品. 菠菜中乙酰差酸还原异构酶的抗体和底物 AHB(2-aceto2-hydroxybutyrate)由法国 R. Dumas 教授馈赠. 其他化学试剂均为国产分析纯.

\subsection{AAIR 核基因的 PCR 扩增及序列分析}

用 DNeasy Plant Mini Kit (Qiagen)从 G2 踠豆的叶片中分离总 DNA. 用引物 1(5'-GGCCGCACAAAAATGGCGGCC-3') 和引物 2(5'- GACATTTTTGGAGAGAGAGGA-3'), 及引物 3(5'-TCCTCTCTCTCCAAAAATGTC-3') 和引物 4(5'-GTTACTACACTGGCGCAACTC-3')分别 $\mathrm{PCR}$ 扩增 $A A I R$ 核基因的 $5^{\prime}$ 和 $3^{\prime}$ 端. 扩增获得的两个 PCR 片段纯化后等比例混合, 先不加引 物 PCR 扩增 10 个循环, 再加人引物 1 和引物 4 继续扩增 30 个循环. 把扩增获得的融合 DNA 克隆到 pGEM-T easy vector (Promega)上测序. 将所得的核基因序列与 cDNA 序列比较分析其 内含子和外显子结构.

\subsection{G2 豌豆顶芽总 RNA 的提取及 Northern 杂交}

分别取短日照和长日照下生长的 G2 踠豆在开花前 1 周和开花后 3 周的顶芽, 用 RNeasy Plant Mini Kit(Qiagen)提取总 RNA. 用 ${ }^{32} \mathrm{P}$ 标记的 $A A I R$ cDNA 为探针根据 Zhu 等人 ${ }^{[12]}$ 的方法 进行 Northern 杂交分析.

\subsection{G2 豌豆顶芽叶绿体蛋白的提取及 Western 杂交}

分别取开花前 1 周和开花后 3 周短日照和长日照下生长的 $\mathrm{G} 2$ 踠豆顶芽约 $10 \mathrm{~g}$, 用液氮冷 冻研磨, 加人 3 倍于叶重的试剂 $1(0.4 \mathrm{~mol} / \mathrm{L}$ 蔗糖, $0.05 \mathrm{~mol} / \mathrm{L}$ Tris- $\mathrm{HCl} \mathrm{pH} \mathrm{7.8,0.002} \mathrm{mol/L}$ EDTA, $0.1 \%$ 颈基乙醇), 制成匀浆, 用 4 层滤纸过滤, 滤液在 $1000 \times g, 4{ }^{\circ} \mathrm{C}$ 下离心 $2 \mathrm{~min}$, 弃去 沉淀, 绿色上清液再在 $4500 \times g, 4^{\circ} \mathrm{C}$ 下离心 $1.5 \mathrm{~min}$, 弃去上悬液, 沉淀即为叶绿体. 向沉淀部 分加人 $10 \mathrm{~mL}$ 试剂 1 , 用玻璃棒使沉淀悬浮(动作轻, 并在 $0 \sim 4{ }^{\circ} \mathrm{C}$ 下操作), 在 $4500 \times g, 4^{\circ} \mathrm{C}$ 下离 心 $1.5 \mathrm{~min}$, 收集沉淀. 用圆头玻璃棒搅匀沉淀, 加人 $5 \mathrm{~mL}$ 试剂 $2(0.05 \mathrm{~mol} / \mathrm{L}$ Tris- $\mathrm{HCl} \mathrm{pH} 8.4$, $0.02 \mathrm{~mol} / \mathrm{L}$ EDTA, $0.1 \%$ 巯基乙醇), 使其悬浮, 在 $10000 \times g, 4{ }^{\circ} \mathrm{C}$ 离心 $15 \mathrm{~min}$, 黄色上清液即 为叶绿体蛋白, 也即酶的粗提液. 保存于 $4{ }^{\circ} \mathrm{C}$ 冰箱中备用. 蛋白浓度用 BioRad protein assay kit(BioRad)测定. 用菠菜中的乙酰羟酸还原异构酶的抗体对所提取的叶绿体蛋白做 Western 杂交 分析, 试验按 ECL Plus Western Blotting Detection Reagents(Amersham Pharmacia)的说明书进行.

\subsection{AAIR 酶活性测定}

以 AHB 为底物, 根据文献 ${ }^{[3]}$ 报道的方法测定粗酶液中的酶活性. 用每分钟单位蛋白氧化 
$\mathrm{NADPH}$ 的纳摩尔数来表示酶活性, 即酶活力单位为: $\mathrm{NADPH}$ 氧化的 $\mathrm{nmol} /(\mathrm{mg}$ 蛋白 $\cdot \min )$.

\section{7 原核表达载体的构建及大肠杆菌功能互补实验}

以克隆有 $A A I R$ 完整 $\mathrm{cDNA}$ 编码区的 $\mathrm{pBS}-A A I R$ 为模板, 用引物 5'-CG-GAATTCGCTAGCGCACTAGGCGCCAC-3' (画线处为 EcoR I 位点) 和 5'-AGGGCGAATTGGGTACCG-3' (来自载体 pBluescript SK 上的序列. PCR 产物含有部分载体序列和一个Xho I 位点, 用 Xho I 酶切后将去除全部载体序列), PCR 扩增获得编码 AAIR 成熟肽段的 cDNA, 纯化后用 EcoR I 和 Xho I 双酶切, 插人到 pGEX-4T-1(Amersham Pharmacia)的相应位点之间, 获得能表 达融合蛋白 GST-AAIR 52 581 的原核表达载体 pGEX-AAIR. 用小量诱导表达证明克隆的正确性 和表达融合蛋白的能力.

将 pGEX-AAIR 转化到 ILVC(E.coli 中的乙酰羟酸还原异构酶)缺陷的 E.coli 菌株 AB1203(E. coli Genetic Stock Center, Yale University)中, 在 LB 培养基(含 $100 \mu \mathrm{g}$ 胺芐青霉素)笁选出转化 子后, 挑取单克隆在缺少分枝氨基酸的 M9-ILV 培养基 (M9 培养基 $+0.04 \%$ Glucose $+0.016 \%$ $\operatorname{Arg}+0.0002 \% \mathrm{VtB} 1)$ 上 $37^{\circ} \mathrm{C}$ 培养, 观察生长情况. 同时把含有正常功能 $I L V C$ 基因的 DH5 在 M9-ILV 培养基上培养, 把只转化空载体 pGEX-4T-1 的 AB1203 分别在 M9 + ILV (M9 培养基 + $0.04 \%$ Glucose $+0.016 \%$ Arg $+0.0002 \% \mathrm{VtB} 1+0.010 \% \mathrm{Val}+0.010 \% \mathrm{Ile}+0.010 \% \mathrm{Leu}$ )和 M9-ILV 培养基上培养进行对照观察.

\section{$1.8{ }^{14} \mathrm{C}$ 标记的分枝氨基酸的产生、纯化、衍生及检测}

从转化 pGEX-AAIR 或 pGEX-4T-1 空载体的 XL1-Blue 新鲜平板挑取单克隆转接到 LB 培 养基中 $37^{\circ} \mathrm{C}$ 过夜培养. 然后按 1：100转接至 $50 \mathrm{~mL} \mathrm{LB}$ 培养基 $\left(100 \mu \mathrm{g} / \mathrm{mL} \mathrm{Amp}\right.$ )中 $37^{\circ} \mathrm{C}$ 培养 至 $A_{600} \approx 0.4$, 取 $12 \mathrm{~mL}$ 菌液转移到 $15 \mathrm{~mL}$ 离心管中 $5000 \times g$ 离心 $5 \mathrm{~min}$ 沉淀细胞. 用 $4 \mathrm{~mL}$ $\mathrm{LB}(100 \mu \mathrm{g} / \mathrm{mL} \mathrm{Amp})$ 充分悬浮细胞并同时加人 $6 \mu \mathrm{Ci}$ 的 ${ }^{14} \mathrm{C}$-丙酮酸和 $\mathrm{IPTG}$ (至终浓度为 1 $\mathrm{mmol} / \mathrm{L}), 37^{\circ} \mathrm{C}$ 继续培养 $6 \mathrm{~h}$ 后收获菌体. 细胞用 $5 \mathrm{~mL} 80 \%$ 的甲醇溶液充分悬浮后于 $-20^{\circ} \mathrm{C}$ 静 置过夜, 然后在室温剧烈振荡 $5 \mathrm{~min}, 1000 \times \mathrm{g}$ 离心 $10 \mathrm{~min}$. 上清转移到蒸馏瓶中, 再用 $5 \mathrm{~mL}$ $80 \%$ 的甲醇溶液悬浮沉淀并剧烈振荡 $5 \mathrm{~min}$, 重复两次, 上清都转人同一个蒸馏瓶中. 蒸馏浓 缩细胞提取液至 $5 \mathrm{~mL}$ 左右, 用 Dowex 50-X4 $\left(\mathrm{H}^{+}\right)$作为离子交换介质 ${ }^{[13]}$, 得到的自由氨基酸溶 液浓缩至 $1 \mathrm{~mL}$ 后用 $0.45 \mu \mathrm{m}$ 过滤器过滤. 根据 Näsholm 等人 ${ }^{[14]}$ 的方法得到衍生氨基酸, 用 Waters Pico-Tag HPLC 系统进行分离, 用 Shimadzu Model RF 530 苂光监测仪检测(激发波长 为 $260 \mathrm{~nm}$, 检测波长为 $313 \mathrm{~nm}$ ). 按 $1 \mathrm{~mL} / \mathrm{min}$ 收集洗脱液, 然后用液闪计数仪检测各组分的放 射性强度.

\subsection{G2 豌豆核蛋白的提取}

分别取在短日照和长日照下生长的 $\mathrm{G} 2$ 踠豆开花前 1 周和开花后 3 周的顶芽组织约 $20 \mathrm{~g}$, 用液氮迅速冷冻后在研钵中研磨成粉末, 后续提取方法根据 Takaiwa 等人 ${ }^{[15]}$ 的方法进行, 用 BioRad protein assay kit (BioRad)测定核蛋白的浓度.

\subsection{0 富 A/T 区探针的制备}

将 3 个富 A/T 区(图 5(a) (c)) 作为凝胶阻滞实验的探针, 先合成两条互补的单链 DNA, 等 摩尔数混合后沸水浴中变性 $5 \mathrm{~min}$, 再自然冷却至室温, 使两条 DNA 链退火形成双链 DNA. 该双链 DNA 片段的两端都有 6 个核苷酸的 $5^{\prime}$ 突出. 在 Klenow 酶(Promega)催化下将 $\alpha-{ }^{32} \mathrm{P}$ $\mathrm{dATP}$ 中的 ${ }^{32} \mathrm{P}$ 标记在 $3^{\prime}$ 端, 经过聚丙烯酰胺凝胶电泳分离回收, 得到用于凝胶阻滞实验的富 
$\mathrm{A} / \mathrm{T}$ 区探针.

\subsection{1 凝胶阻滞实验}

为了减少非特异性的 DNA-protein 结合, 先在 $15 \sim 20 \mu \mathrm{L}$ 结合缓冲液 $(10 \mathrm{mmol} / \mathrm{L}$ Tris- $\mathrm{HCl}$ $\mathrm{pH}$ 8.0, $1 \mathrm{mmol} / \mathrm{L} \mathrm{EDTA}, 1 \mathrm{mmol} / \mathrm{L} \mathrm{DTT}, 50 \mathrm{mmol} / \mathrm{L} \mathrm{NaCl}$ 和 $5 \%$ 甘油)中加人 $5 \mu \mathrm{g} \mathrm{G} 2$ 踠豆核蛋 白, $1 \mu \mathrm{g}$ 双链 poly (dI-dC) 和 $2 \mu \mathrm{g}$ 非特异的小牛胸腺 $\mathrm{DNA}$ (超声波破碎至 $1 \mathrm{~kb}$ 左右), $25^{\circ} \mathrm{C}$ 静置 $10 \mathrm{~min}$. 再加人 $1 \mathrm{ng}{ }^{32} \mathrm{P}$ 标记的富 $\mathrm{A} / \mathrm{T}$ 区探针, 充分混合后 $25^{\circ} \mathrm{C}$ 温育 $20 \mathrm{~min}$. 总反应体系约为 20 30 $\mu \mathrm{L}$. 将反应混合物上样于经预电泳的 $8 \%$ 的聚丙烯酰胺凝胶, 电泳缓冲液为 $0.5 \times \mathrm{TBE}$ (54 mmol/L Tris-borate $\mathrm{pH} 8.3,1 \mathrm{mmol} / \mathrm{L}$ EDTA), $10 \mathrm{~V} / \mathrm{cm}$ 电泳 $2 \sim 3 \mathrm{~h}$. 将电泳完毕后的凝胶在 $10 \%$ 冰醋酸中固定 $10 \mathrm{~min}$, 然后真空抽干. 用 $\mathrm{X}$ 线片于 $-70^{\circ} \mathrm{C}$ 放射自显影 $1 \sim 3 \mathrm{~d}$.

\section{2 结果}

\section{$2.1 A A I R$ 核基因的克隆及序列分析}

为了保证 PCR 的效率, 首先用 PCR 的方法分别扩增出 $A A I R$ 基因的 $5^{\prime}$ 和 $3^{\prime}$ 端, 然后用 PCR 的方法把两个 PCR 片段融合在一起获得 $3337 \mathrm{bp}$ 的 DNA(图 1). 与以前克隆的 AAIR cDNA 序 列比较分析表明, $A A I R$ 的核基因由 9 个外显子和 8 个内含子组成. AAIR cDNA 的 EMBL Bank 登录号为 Y17796, 核 DNA 的登录号为 AJ251333.

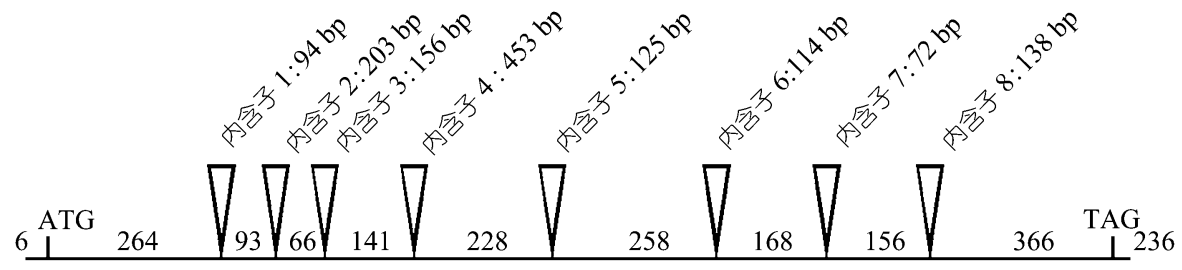

图 1 AAIR 的基因结构示意图

核基因全长 $3337 \mathrm{bp}$, 共有长度分别为 $94,203,156,453,125,114,72$ 和 $138 \mathrm{bp}$ 的内含子 8 个, 开放读码框全长 $1740 \mathrm{bp}$

\subsection{AAIR 基因表达特性分析}

用 Northern 杂交和 Western 杂交分析 AAIR 在长日照和短日照下生长的 G2 踠豆开花前后 的表达情况, 发现在短日照下 AAIR 的表达不受生殖生长的影响, 开花前后都维持较高的表达, 而在长日照下 AAIR 的表达在开花后迅速下降(图 2).

\section{$2.3 \mathrm{AAIR}$ 酶活性分析}

对长、短日照下生长的 G2 踠豆在不同生长时期顶芽部位的酶活性进行了分析(图 3). 结 果显示, 在短日照下 AAIR 的酶活性在开花后有所提高, 而在长日照下 AAIR 的酶活性在开花 后则迅速下降，与 Northern 杂交和 Western 杂交结果相一致.

\section{4 豌豆 AAIR 基因在大肠杆菌中的表达及活性分析}

$A A I R$ 在大肠杆菌中的同源基因为 $I L V C$, 即乙酰差酸还原异构酶. 为了验证 AAIR 是否确 实具有乙酰差酸还原异构酶酶促活性, 首先用 ILVC 缺陷的大肠杆菌 AB1203 菌株做了功能互 补实验, 结果如图 4(a). 由于乙酰差酸还原异构酶的缺陷, AB1203 菌株不能合成分枝氨基酸, 所以不能在没有外加 Leu, Val 和 Ile 的培养基上生长(左下). 而当把能诱导表达 GST-AAIR ${ }_{52 \sim 581}$ 融合蛋白的 pGEX-AAIR 转人 AB1203 菌株后, 在 IPTG 诱导表达条件下能在无分枝氨基酸的 
M9 培养基上生长(右下), 显示 GST-AAIR ${ }_{52 \sim 581}$ 可能具有乙酰羟酸还原异构酶的活性.

为了用同位素标记法进一步分析 AAIR 的 酶活性，首先分析了标准分枝氨基酸的洗脱时 间. Val, Leu 和 Ile 与 FMOC-Cl 反应后的衍生物 经过 HPLC 分离后苂光检测结果显示, 3 种分枝 氨基酸在 16 18 min 之间依次洗脱下来(图 4(b)). 用 ${ }^{14} \mathrm{C}$ 标记的丙酮酸处理表达 GST-AAIR 融合 蛋白的 XL1-Blue 和只表达 GST 的对照菌株, 对 其分枝氨基酸合成能力的分析显示, 在 IPTG 诱 导条件下, 经 ${ }^{14} \mathrm{C}$ 标记的丙酮酸处理 $6 \mathrm{~h}$ 后, 转 化 pGEX-AAIR 的 XL1-Blue 比转化 pGEX-4T-1 的对照菌株细胞中的被标记的分枝氨基酸有显 著的增加, 表明表达 GST-AAIR 52 581 的菌株比 只表达 GST 的对照菌株合成分枝氨基酸的能力 有显著提高(图 4(c)).

\section{5 内含子中的富 $\mathrm{A} / \mathrm{T}$ 区及其与 G2 豌豆核 蛋白的结合实验}

对内含子序列分析发现, 第 3 5 个内含子 中都各含有特殊的富 $\mathrm{A} / \mathrm{T}$ 区序列, 其中第 3 个 内含子中的富 $\mathrm{A} / \mathrm{T}$ 区由 $12 \mathrm{bp}$ 的正向重复序列

\begin{tabular}{|c|c|c|c|}
\hline \multicolumn{2}{|c|}{ 开花的 } & & \\
\hline SD & LD & SD & LD \\
\hline & & & \\
\hline
\end{tabular}
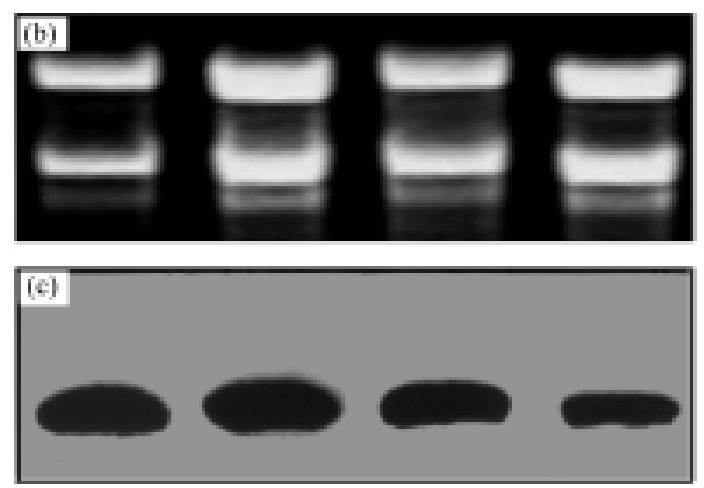

图 2 Northern 杂交和 Western 杂交分析 AAIR 在 长日照(LD)和短日照(SD)下生长的 $\mathrm{G} 2$ 踠豆开花 前后的表达情况

(a) AAIR mRNA 的 Northern 杂交结果; (b) 总 RNA 中的 $26 \mathrm{~S}$ 和 18S rRNA 条带, 表明各泳道中的上样量相同; (c) Western 分析不同生长时期踠豆植株中 AAIR 蛋白质的丰度. 开花 前, 开花前 1 周的顶芽, 首花后 3 周, 第一朵花开花后 3 周 的顶芽 组成, 间隔 $7 \mathrm{bp}$ (图 5(a)); 第 4 个内含子中的富 $\mathrm{A} / \mathrm{T}$ 区由 $7 \mathrm{bp}$ 的正向重复序列组成, 间隔 $8 \mathrm{bp}$ (图 5(b)); 第 5 个内含子中的富 $\mathrm{A} / \mathrm{T}$ 区由 $14 \mathrm{bp}$ 的对称重复序列组成, 间隔 $7 \mathrm{bp}$ (图 5(c)).

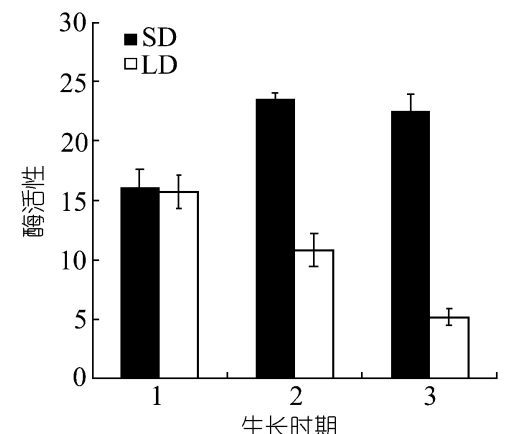

图 3 对短日照(SD)和长日照(LD)下 生长的 $\mathrm{G} 2$ 踠豆在不同时期顶芽部位 的酶活性分析

1. 开花前 1 周的顶芽; 2. 第一朵花刚开放时 的顶芽; 3. 第一朵花开花后 3 周的顶芽. 酶活 性用 $\mu \mathrm{mol} /(\mathrm{L} \mathrm{NADP} \cdot \mathrm{mg}$ 总蛋白・ min)表示

因为这种特殊的对称结构很可能是基因内部的调控 序列, 所以用凝胶阻滞实验分析了 3 个富 $\mathrm{A} / \mathrm{T}$ 区与短日 照和长日照下的 $\mathrm{G} 2$ 踠豆核蛋白结合情况. 初步研究表明, 3 个富 $\mathrm{A} / \mathrm{T}$ 区都能与核蛋白中的某些蛋白组分特异结合, 这些富 $A / T$ 区结合蛋白在短日照条件下的表达不受发育 调控, 而在长日照下开花后迅速减少. 图 5(d)显示用第 5 个内含子中的富 $\mathrm{A} / \mathrm{T}$ 区做探针进行凝胶阻滞实验所获得 的结果.

\section{3 讨论}

乙酰羟酸还原异构酶在生物的发育过程中具有高度 的保守性, 特别是在 4 个功能区保守性更高 ${ }^{[2]}$. 在大肠杆 菌的 ILVC 和 AAIR 之间全蛋白的同源性还是很低的, 但 是在功能区内重要的氨基酸部位都保持非常高的保守性 

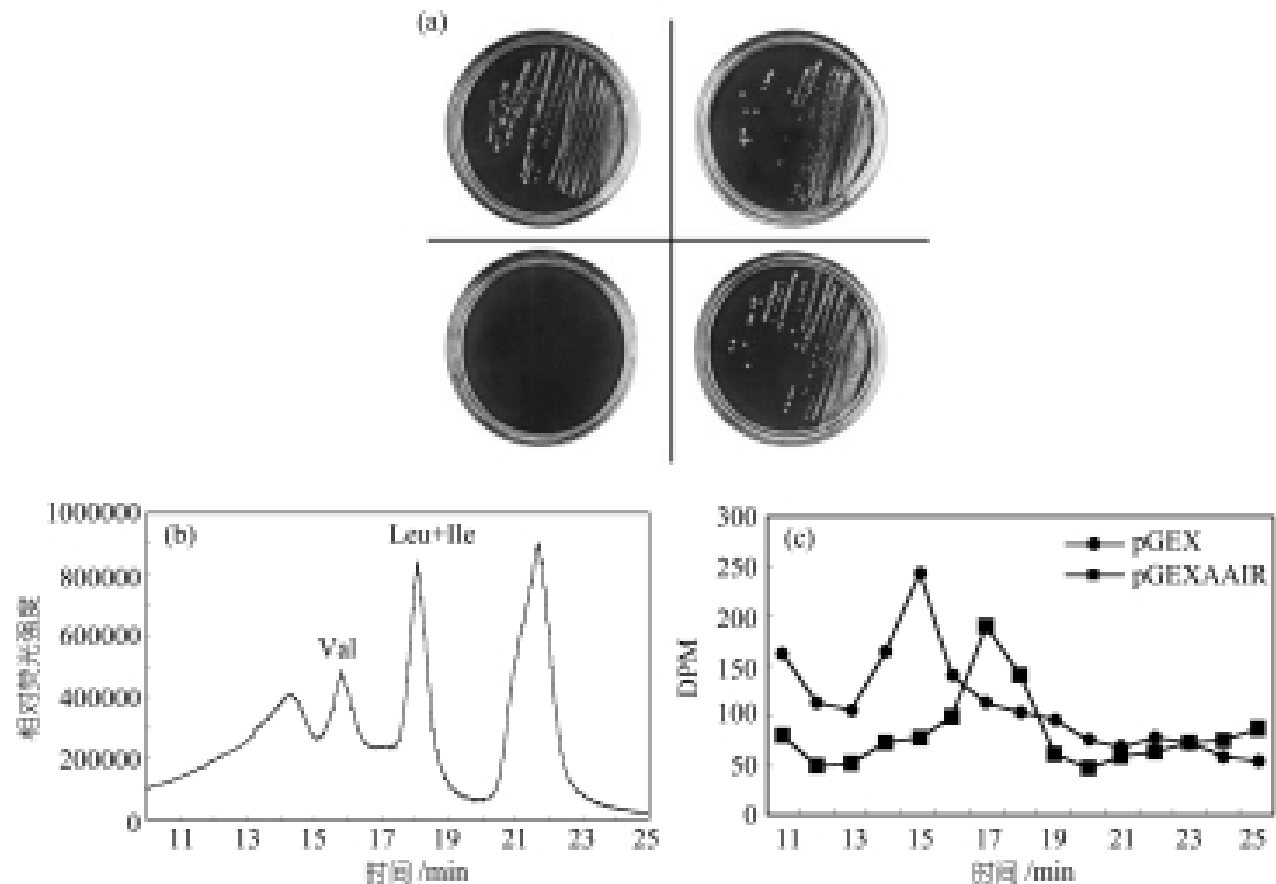

图 4 AAIR 在 E. coli 中的酶活性分析

(a) AAIR 在其同源基因 ILVC 缺陷型大肠杆菌 AB1203 中的功能互补实验. 左上, DH5 菌株正对照. 右上, AB1203 菌株在 M9+ILV 培养基上可正常生长. 左下, AB1203 菌株在 M9-ILV 培养基上不能生长. 右下, 转人 pGEX-AAIR 质粒的 AB1203 菌 株在 IPTG 的诱导下可在 M9-ILV 培养基上正常生长. (b) 标准分枝氨基酸分析, 即 Val, Leu 和 Ile 与 FMOC-Cl 反应后的衍生 物经过 HPLC 分离后检测结果. (c) 转化 pGEX-AAIR 的 XL1-Blue 和转化 pGEX-4T-1 的对照菌株经 ${ }^{14} \mathrm{C}$ 标记的丙酮酸处理 $6 \mathrm{~h}$ 后的游离氨基酸放射强度分析

(结果未显示). 当在乙酰羟酸还原异构酶缺陷的大肠杆菌中表达 G2 踠豆同源基因的时候，该 缺陷型菌株即恢复了合成分枝氨基酸的功能, 不但证明了 AAIR 的生物活性, 而且表明在高等 植物叶绿体中的乙酰羟酸还原异构酶能够在原核生物的细胞质内行使正常的生物功能(图 4). 同位素标记实验进一步支持了这一结论. 值得注意的是, 实验中所表达的是 GST 与 AAIR 成 熟肽段的融合蛋白. 很显然, GST 的存在并没有抑制 AAIR 酶活性的发挥，暗示 AAIR 在高级 结构上的高度自主性.

Feo 等人 ${ }^{[16] 、 P a s s a n t i n o ~}$ 等人 $^{[17]}$ 首次报道了富 A/T 区在基因表达调控中的作用. 该元件存 在于一个与人体 $\beta$-烯醇化酶( $\beta$-Enolase, ENO-3)转录调节相关的内含子中, 它通过与肌细胞 特异增强子因子 2 (myocyte-specific enhancer factor 2)相结合来调节基因的表达. 植物中也发 现了富 $\mathrm{A} / \mathrm{T}$ 区的特殊调节作用 ${ }^{[18]}$. 有意思的是, $A A I R$ 基因内部的富 $\mathrm{A} / \mathrm{T}$ 区都是有对称性的二 聚体, 而前人所报道的富 $\mathrm{A} / \mathrm{T}$ 区仅有这些富 $\mathrm{A} / \mathrm{T}$ 区的一半, 因此, 这些富 $\mathrm{A} / \mathrm{T}$ 区的功能可能更 为强大. 凝胶阻滞实验显示 $A A I R$ 内含子的富 $\mathrm{A} / \mathrm{T}$ 区能够与 $\mathrm{G} 2$ 豌豆核蛋白中特定蛋白组分相 结合, 而且结合能力很强. 这种富 A/T 区结合蛋白在 G2 踠豆短日照不衰老的植株中保持一个 稳定的表达模式，在开花前和开花后都没有明显的差异(图 5(d)). 在长日照条件下，这些结合 蛋白的含量在开花前与短日照下相近, 但在开花后 3 周即有大幅度的下降. 因此, 这些富 $\mathrm{A} / \mathrm{T}$ 区结合蛋白可能是受光周期调控的反式作用元件，它们的高表达与 G2 豌豆的旺盛的营养生长 
(a)

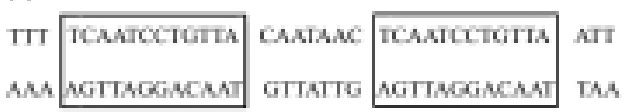

(b)

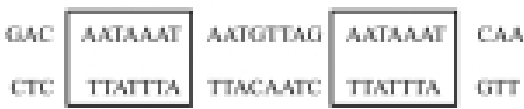

(c)
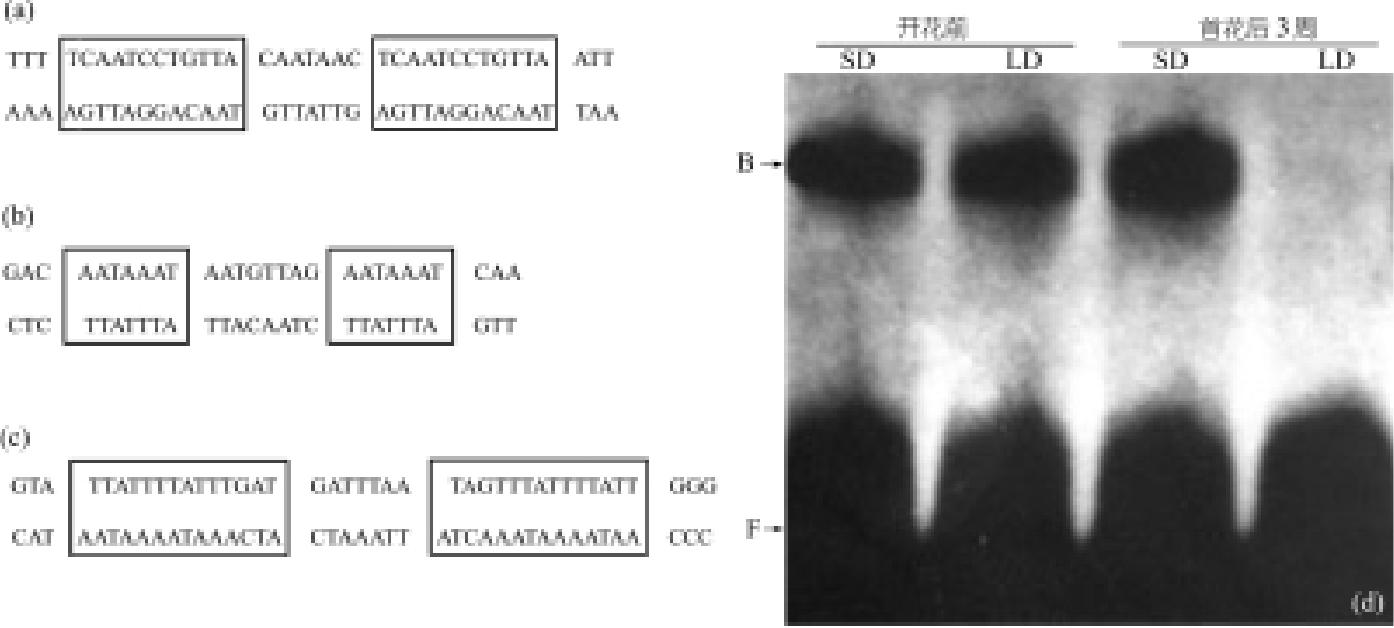

图 $5 A A I R$ 基因内含子中的富 $\mathrm{A} / \mathrm{T}$ 区及其与 $\mathrm{G} 2$ 踠豆核蛋白的结合分析

(a) 第 3 个内含子中的富 $\mathrm{A} / \mathrm{T}$ 区由 $12 \mathrm{bp}$ 的正向重复序列组成, 间隔 $7 \mathrm{bp}$. (b) 第 4 个内含子中的富 $\mathrm{A} / \mathrm{T}$ 区由 $7 \mathrm{bp}$ 的正向重 复序列组成, 间隔 $8 \mathrm{bp}$. (c) 第 5 个内含子中的富 $\mathrm{A} / \mathrm{T}$ 区由 $14 \mathrm{bp}$ 的对称重复序列组成, 间隔 $7 \mathrm{bp}$. (d) 用第 5 个内含子中的 富 $\mathrm{A} / \mathrm{T}$ 区做探针与 $\mathrm{SD}$ 和 $\mathrm{LD}$ 条件下的 G2 踠豆核蛋白进行凝胶阻滞实验. F 示游离探针, B 示探针与蛋白之间形成的结合滞 后条带. 时期标注同图 2

相对应, 并且这种表达不受生殖生长的影响，所以这些蛋白很可能是抑制植物进人衰老过程 的重要调控因子.

分析短日照和长日照生长条件下生长的 G2 踠豆顶芽发现, 在短日照条件下, 无论是营养 生长阶段还是生殖生长阶段, AAIR 基因都保持较高的表达强度和酶活性(图 2 和 3). 在长日照 条件下, $A A I R$ 转录产物的丰度和酶活性在开花后 3 周就显著降低. 由于分枝氨基酸生物合成途 径中几个相关酶在发育中的幼嫩组织里均有很高的丰度和酶活性 ${ }^{[19 ~ 24]}$, 我们推测 $A A I R$ 基因的 表达有可能与 G2 踠豆在短日照条件下的持续旺盛生长有关.

参考文献

1 朱玉贤, 张翼风, 李慧英. 用 cDNA 差示分析法克隆受 GA 抑制的踠豆基因. 中国科学, C 辑, 1997, 27(3): 253 257

2 徐华松, 徐云剑, 顾雪松, 等. 䙵豆乙酰羟酸还原异构酶基因 cDNA 克隆及其表达. 科学通报, 2001, 46 (12): 1022 1025

3 Dumas R, Joyard J, Douce R. Purification and characterization of acetohydroxyacid reductoisomerase from spinach chloroplasts. Biochem J, 1989, 262: 971 976

4 Dumas R, Butikofer M C, Job D, et al. Evidence for two catalytically different magnesium binding sites in acetohydroxy acid isomeroreductase by site-directed mutagenesis. Biochemistry, 1995, 34: 6026 6036

5 Singh B K. Biosynthesis of valine, leucine, and isoleucine. In: Singh B K, ed. Plant Amino Acids-Biochemistry and Biotechnology. New York: Marcel Dekker Inc, 1999. 227 247

6 Reynolds T L. Effect of chlorsulfuron valine and isoleucine on division and tracheary element differentiation in cell suspension cultures of Solanum carolinense. J Plant Physiol, 1986, 125(1-2): 179 184

7 Spackman V M T, Cobb A H. Cell cycle inhibition of potato root tips treated with imazethapyr. Annals of Applied Biology, 1999, 135(3): 585 587

8 Zelenaya-Troitskaya O, Perlman P S, Butow R A. An enzyme in yeast mitochondria that catalyzes a step in branched-chain amino acid biosynthesis also functions in mitochondrial DNA stability. EMBO J, 1995, 14: 3268 3276 
9 MacAlpine D M, Perlman P S, Butow R A. The numbers of individual mitochondrial DNA molecules and mitochondrial DNA nucleoids in yeast are co-regulated by the general amino acid control pathway. EMBO J, 2000, 19(4): 767 775

10 Kaufman B A, Newman S M, Hallberg R L, et al. In organello formaldehyde crosslinking of proteins to mtDNA: Identification of bifunctional proteins. Proc Natl Acad Sci USA, 2000, 97: 7772 7777

11 Mazliak P. Plant membrane lipids: Changes and alterations during aging and senescence. In: Lieberman M, ed. Post-harvest. Physiology and Crop Preservation. NATO Advanced Study Institutes Series. New York: Plenum Press, 1983. 123 140

12 Zhu Y, Zhang Y, Luo J, et al. PPF-1, a post-floral-specific gene expressed in short-day-grown G2 pea, may be important for its never-senescing phenotype. Gene, 1998, 208: 1 6

13 Thompson J F, Morris C J, Gering R K. Purification of plant amino acids for paper chromatography. Analytical Chemistry, 1959, 31: 1028 1031

14 Näsholm T, Sandberg G, Ericsson A. Quantitative analysis of amino acids in conifer tissues by high-performance liquid chromatography and fluorescence detection of their 9-fluorenylmethyl chloroformate derivatives. Journal of Chromatography, 1987, 396: 225 236

15 Takaiwa F, Oono K. Interaction of an immature seed-specific transacting factor with the 5'- upstream region of a rice glutelin gene. Mol Gen Genet, 1990, 224(2): 289 293

16 Feo S, Antona V, Barbieri G, et al. Transcription of the human beta enolase gene (ENO-3) is regulated by an intronic muscle-specific enhancer that binds myocyte-specific enhancer factor 2 proteins and ubiquitous G-rich-box binding factors. Mol Cell Biol, 1995, 15(11): 5991 6002

17 Passantino R, Antona V, Barbieri G, et al. Negative regulation of beta enolase gene transcription in embryonic muscle is dependent upon a zinc finger factor that binds to the G-rich box within the muscle-specific enhancer. J Biol Chem, 1998, 273(1): 484 494

18 Lumbreras V, Stevens D R, Purton S. Efficient foreign gene expression in Chlamydomonas reinhardtii mediated by an endogenous intron. Plant J, 1998, 14 (4): 441 447

19 Wiersma P A, Schmiemann M G, Condie J A, et al. Isolation, expression and phylogenetic in-heritance of an acetolactate synthase gene from Brassica napus. Mol Gen Genet, 1989, 219: 413 420

20 Keeler S J, Sanders P, Smith J K, et al. Regulation of tobacco acetolactate synthase gene expression. Plant Physiol, 1993, 102: $1009 \sim 1018$

21 Singh B K, Newhouse K E, Stidham M A, et al. Actohydroxyacid synthase-imidazolinone interaction. In: Barak Z, Chipman D M, Schloss J V, eds. Biosynthesis of Branched Chain Amino Acids. Weinheim: VCH, 1990. 357 372

22 Stidham M A, Singh B K. Imidazolinone-acetohydroxyacid synthase interactions. In: Shaner L L, O'Connor S L, eds, the lmidazolinone Herbicides. Boca Raton: CRC Press, 1991. 71 90

23 Szamosi I T, Shaner D L, Singh B K. Identification and characterization of a biodegradative form of threonine deghdratase in senescing tomato leaf. Plant Physiol, 1993, 101: 999 1004

24 Hofgen R, Laber B, Schuttke I, et al. Repression of acetolactate synthase activity through antisense inhibition: Molecular and biochemical analysis of transgenic potato (Solanum tuberosum L. cv Desiree) plants. Plant Physiol, 1995, 107: 469 477 